\title{
ANÁLISE DOS MODOS DE TRANSFERÊNCIA DE CALOR EM UM CONJUNTO MECÂNICO COMPOSTO POR UM ROLO DE EXTRAÇÃO E MANCAIS DE CONTATO ROLANTE*
}

Pedro Henrique Vasconcellos Apipe ${ }^{1}$

\section{Resumo}

O objetivo do trabalho consistiu em estudar os modos de transmissão de calor existentes em um conjunto mecânico composto por um rolo de extração e mancais de contato rolante em decorrência do movimento de um tubo laminado sobre o mesmo na planta da Vallourec Brasil. A motivação para o estudo surgiu na avaliação de como os fenômenos térmicos presentes resultariam na distribuição de temperatura através da superfície do conjunto e qual a sua relação com a disponibilidade desse equipamento. Para isso a análise desenvolvida foi baseada no emprego da dinâmica dos fluidos computacionais (CFD), associada a imposições subsidiárias durante a definição das condições de contorno do problema. Como resultado a distribuição de temperatura através da superfície do conjunto foi determinada, sobretudo na pista interna do rolamento e na região na qual se encontra o sistema de refrigeração. Desse modo conclui-se que embora o sistema de refrigeração dissipe uma grande quantidade de calor a sua eficiência não é satisfatória. Fato esse descrito pela presença de temperaturas superiores as necessárias para a ocorrência do fenômeno de ponto de gota.

Palavras-chave: Transmissão de calor; Conjunto mecânico; CFD.

\section{MODES ANALYSIS OF HEAT TRANSFER IN A MECHANICAL JOINT COMPOUND FOR A ROLL OF EXTRACTION AND ROLLING CONTACT BEARINGS}

\section{Abstract}

The objective of this work was to study the modes of heat transfer in a mechanical assembly consisting of a roll of extraction and rolling contact bearings due to the motion of a tube on the same in the Vallourec Brazil plant. The motivation for the study arose in the evaluation of how the thermal phenomena result in the present temperature distribution across the surface of the assembly and what is its relationship to the availability of such equipment. The analysis developed was based on the use of computational fluid dynamics (CFD) in association with subsidiaries charges to define the boundary conditions of the problem. As a result the temperature distribution across the array surface was determined mainly on the inner race of the bearing and in the region that there is the cooling system. This way was possible to conclude that even though the cooling system dissipate a large amount of heat the efficiency of cooling system is not satisfactory. A fact this described by the presence of temperatures higher than the necessary for the occurrence of the phenomenon of dropping point.

Keywords: Heat transfer; Mechanical assembly; CFD

1 Engenharia Mecânica, Engenheiro, Laminação/Têmpera, Vallourec e Sumitomo Tubos do Brasil (VSB), Belo Horizonte, Minas Gerais, Brasil.

* Contribuição técnica ao $35^{\circ}$ Seminário de Balanços Energéticos Globais e Utilidades e $29^{\circ}$ Encontro de Produtores e Consumidores de Gases Industriais, 13 a 15 de agosto de 2014, São Paulo, SP, Brasil. 


\section{INTRODUÇÃO}

\subsection{Objetivos}

O objetivo do trabalho proposto visa identificar como os mecanismos de transmissão de calor, condução, convecção e radiação térmica atuantes no conjunto mecânico (rolo de extração e mancais de contato rolante) repercutem na distribuição de temperatura ao longo de toda a superfície do sistema. Distribuição de temperatura essa a ser verificada devido a suspeita da existência de elevados níveis de temperatura em partes funcionais do sistema estritamente ligadas a sua disponibilidade na área produtiva. Com isso objetiva-se compreender a possível relação existente entre a disponibilidade deste sistema para uma situação crítica de serviço e a eficiência da refrigeração atualmente empregada nesse conjunto. Por isso o foco da pesquisa é também baseado no conhecimento de como os efeitos térmicos podem repercutir no bom funcionamento dos mancais e da funcionalidade do lubrificante. Funcionalidade essa descrita como ponto de gota da graxa, uma vez que a graxa é o lubrificante utilizado nesse sistema.

\subsection{Referências Bibliográficas.}

\subsubsection{Mancais de contato rolante}

Ponto importante a ser avaliado para que o mancal de contato rolante apresente um adequado funcionamento consiste na correlação existente entre o sistema de lubrificação empregado (graxa ou óleo) e a temperatura de funcionamento do conjunto. De modo que a presença de temperaturas de trabalho elevadas podem danificar os rolamentos existentes, sendo a lubrificação ineficiente ou instalação imprópria desse componente exemplo de possíveis causas de aumento de temperatura e conseqüentemente falha do sistema [1]. Associado a esse aumento de temperatura provocado por uma lubrificação ineficiente Stouterau [2] propõe que existe uma restrita correlação entre o aquecimento dos mancais quando lubrificados com graxa e uma dada razão de rotações. No que refere-se às causas dos efeitos provocados pelo excesso de temperatura no sistema Carreteiro, Belmiro [3] citam os intervalos de relubrificação em mancais cujo lubrificante empregado é a graxa, por meio de gráficos empregados pelos fabricantes de rolamentos, com base em uma análise de rotação do sistema, afim de evitar o desgaste excessivo de componentes desse sistema.

Segundo Burmeister [4] ponto importante a ser avaliado na transferência de calor entre os corpos rolantes de um mancal, surge na existência de um fluxo conhecido como escoamento de Coutte. Escoamento esse definido caso se tenha a ocorrência de duas superfícies paralelas em movimento relativo separadas por um meio fluido. Burmeister [4] propõe assim, que uma vez tal ocorrência esteja presente no sistema o fluxo de calor existente entre essas superfícies pode então ser calculado, uma vez que sejam conhecidas a condutividade térmica do fluído existente ente as superfícies, a distância entre as mesmas, o valor da temperatura da superfície inferior e a temperatura adiabática do sistema.

\subsubsection{Lubrificantes}

Segundo Carreteiro, Belmiro [3] os lubrificantes podem ser definidos como graxas ou óleos. De acordo com esses autores temos para a graxa a seguinte definição: "É uma combinação de um fluido com um espessante, resultando de um produto

* Contribuição técnica ao $35^{\circ}$ Seminário de Balanços Energéticos Globais e Utilidades e $29^{\circ}$ Encontro de Produtores e Consumidores de Gases Industriais, 13 a 15 de agosto de 2014, São Paulo, SP, Brasil. 
homogêneo com qualidades lubrificantes" [3]. Tais lubrificantes são compostos por um óleo base, um espessante e outros agentes que podem ser capazes de conferir certas características e propriedades especiais como no caso de aditivos. A função do óleo base é a de conferir as propriedades lubrificantes da graxa, já a função do espessante, os quais geralmente são sabões metálicos de Sódio, Cálcio, Alumínio, Lítio e sabão complexo está estritamente ligada ao ponto de gota da graxa [5]. Ponto de gota esse definido de acordo com Carreteiro, Belmiro [3] como a temperatura na qual o produto (graxa) se torna fluido.

A importância do ponto de gota na seleção de uma graxa consiste na especificação da faixa de temperaturas, a qual a graxa pode resistir em seu regime de trabalho sem perder suas propriedades lubrificantes [1].

\subsubsection{Modos de transmissão de calor}

Segundo Incropera et al. [6] definimos transmissão de calor como: energia térmica em transito devido a uma diferença de temperatura no espaço, as quais podem ocorrer de 3 maneiras (Condução, Convecção e Radiação Térmica) de acordo com os mecanismos e meios existentes para a sua transferência.

A respeito da análise de problemas de condução de calor sob uma ótica unidimensional para Kreith, Bohn [7] muitos problemas práticos nos quais a temperatura e o fluxo de calor são tratados como função de uma única variável podem ser aceitáveis, desde que algumas condições sejam satisfeitas. Kreith, Bohn ${ }^{7}$ propõem que tais condições são satisfeitas caso os limites de um sistema não sejam irregulares e a temperatura ao longo desses limites seja uniforme. Uma vez que tais condições não sejam satisfeitas os mesmos afirmam ser 0 tratamento unidimensional da condução de calor não satisfatório. Tal fato deve-se a distribuição de temperatura ser possivelmente uma função de duas ou três coordenadas. Desse modo Kreith, Bohn [7] afirmam que a condução de calor pode ser tratada pela análise de problemas bidimensionais ou tridimensionais os quais podem ser resolvidos por métodos analíticos, gráficos e numéricos.

De acordo com Siegel, Howell [8] no que refere-se a um sistema em que estejam envolvidos os fenômenos de radiação térmica e condução a formulação matemática proposta para a abordagem desse problemas envolve o tratamento desses como sistemas desacoplados ou acoplados. Sistemas assim descritos como desacoplados caso a propriedade de interesse em análise (fluxo térmico) possa ser quantificada determinando os efeitos da condução e convecção separados e por fim adicionando os mesmos. Já para sistemas que sejam descritos como acoplados a mesma abordagem não pode ser evidenciada, uma vez que a equação governante para resolução do problema precisa ser resolvida com a presença dos dois modos de transferência de calor descritos simultaneamente. Segundo Siegel, Howell [8] na presença da combinação entre radiação e convecção a formulação matemática proposta é similar a descrita para problemas que envolvam radiação e condução. A diferença surge na presença da diferença de temperatura que governa a convecção no lugar das derivadas governantes da formulação proposta para a condução. Nesse tipo de problema a formulação proposta é comumente empregada tratando o sistema como não linear, logo acoplado. Já para sistemas que apresentem os três fenômenos, radiação, condução e convecção envolvidas na transferência de calor a formulação proposta como salienta Siegel, Howell [8] para a solução torna-se extremamente difícil. Tal fato explica-se pela presença do acréscimo da diferença de temperatura proveniente da convecção, assim como das derivadas da temperatura provenientes da condução na equação de energia proposta para a solução do

* Contribuição técnica ao $35^{\circ}$ Seminário de Balanços Energéticos Globais e Utilidades e $29^{\circ}$ Encontro de Produtores e Consumidores de Gases Industriais, 13 a 15 de agosto de 2014, São Paulo, SP, Brasil. 


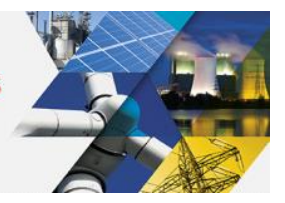

problema. Associada a essa dificuldade surge também a presença de um grande número de parâmetros independentes originários das quantidades que governam os fenômenos de condução e convecção. Como resultado da complexidade assim envolvida em problemas em que estejam envolvidos os três mecanismos de transmissão de calor, a não existência de soluções clássicas e métodos de solução é comumente encontrada. De modo que a maioria das soluções obtidas é determinada por meio de técnicas numéricas.

\section{MATERIAIS E MÉTODOS}

A respeito dos métodos utilizados para avaliação do perfil de temperatura no sistema uma metodologia descrita em três etapas foi adotada. As quais foram enunciadas durante o desenvolvimento do trabalho como: modelagem do problema, aquisição de dados e implementação computacional (CFD). No que tange a modelagem do problema foi adotado a criação de um modelo integral simplificado dos mecanismos de transmissão de calor existentes no sistema em uma situação de trabalho crítica, ou seja, a permanência de um tubo incandescente promovendo um fluxo de calor radiante constante sobre a superfície do rolo após um regime de trabalho intermitente do mesmo. Para isso foi utilizado um balanço de energia no sistema em associação com as equações básicas da transferência de calor por radiação e convecção sob condições de escoamento externo e interno em uma geometria cilíndrica estacionária, na qual o emprego de tais equações foi descrito por meio da criação de regiões térmicas no conjunto mecânico em questão. Já a segunda etapa foi baseada na aquisição das grandezas referentes à temperatura da superfície do rolo e dos mancais, temperatura da superfície do tubo, vazão volumétrica existente no sistema, temperatura de entrada da água do sistema de refrigeração, assim como a temperatura da mesma na saída desse sistema. Por fim a terceira etapa baseouse na implementação de um modelo computacional para o sistema com o intuito de avaliar como os efeitos térmicos existentes no sistema descreviam o perfil de temperatura no conjunto mecânico.

\subsection{Regiões Térmicas e Modelo Integral}

Para modelagem do problema as seguintes regiões térmicas apresentadas pela (Figura 1) que descreve a geometria do conjunto mecânico estudado (rolo de extração e mancais de contato rolante) foram definidas na criação do modelo integral.

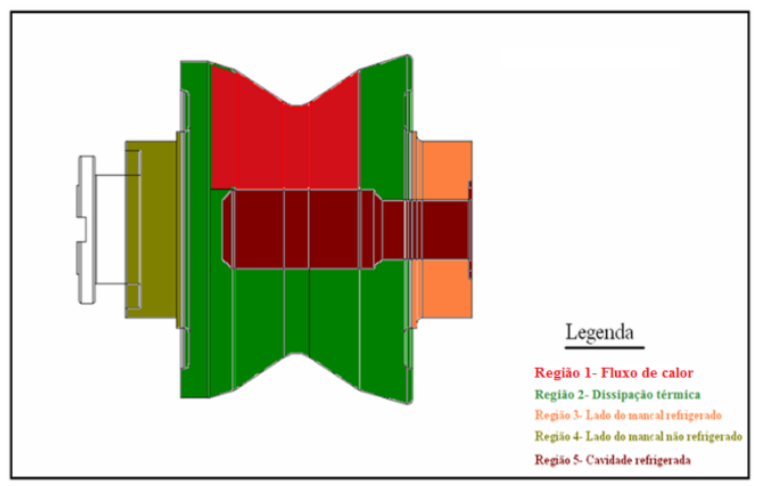

Figura 1: Regiões Térmicas (Modelo Integral)

* Contribuição técnica ao $35^{\circ}$ Seminário de Balanços Energéticos Globais e Utilidades e $29^{\circ}$ Encontro de Produtores e Consumidores de Gases Industriais, 13 a 15 de agosto de 2014, São Paulo, SP, Brasil. 


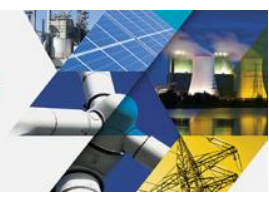

$\mathrm{Na}$ qual o modelo adotado para a região 1 apresentada pela Figura 1 foi descrito pela (Equação 1):

$$
\dot{E}_{\text {entrada }}=\dot{E}_{\text {incidente }}-\dot{E}_{\text {dissipado }}
$$

Em que a energia incidente é definida pelas equações 2 e 3 respectivamente e o fator de forma apresentado pela equação 3 é proposto por Feingold, Gupta [9] para um retângulo infinitamente longo sob uma geometria circular:

$$
\begin{array}{r}
\dot{E}_{\text {incidente }}=q^{\prime \prime}{ }_{\text {Radiação }} \text { Tubo-Rolo } \\
q_{\text {Radiação }}^{\prime \prime}{ }_{\text {Tubo-Rolo }}=\sigma F_{\text {tubo-rolo }}\left(T_{\text {tubo }}{ }^{4}-T_{\text {sup-rolo }}{ }^{4}\right)
\end{array}
$$

A respeito do modelamento da energia dissipada o mesmo é representado pela equação 4, em que os valores do fluxo de calor por radiação entre o rolo e 0 ambiente e o fluxo de calor por convecção foram descritos pela (Equação 5) e (Equação 6) respectivamente.

$$
\begin{array}{r}
E_{\text {dissipado }}=q^{\prime \prime}{ }_{\text {Radiação }}{ }_{\text {Rolo-Ambiente }}+q^{\prime \prime}{ }_{\text {Convecção }} \\
q_{\text {Radiação }}^{\prime \prime}{ }_{\text {Rolo-Ambiente }}=\sigma \varepsilon_{\text {Rolo }}\left(T_{\text {sup-rolo }}{ }^{4}-T_{\infty}{ }^{4}\right) \\
q^{\prime \prime}{ }_{\text {Convecção }}=\bar{h}\left(T_{\text {sup }}-\right.\text { rolo } \\
\end{array}
$$

Assim no que diz respeito ao conhecimento do parâmetro $(\bar{h})$, tanto a convecção forçada como a natural foram avaliadas para a verificação ou não da existência de uma convecção combinada. Para análise da convecção forçada a correlação descrita por Churchil, Bernstein [10] para situações de escoamento forçado em geometria cilíndricas estacionárias foi utilizada. Enquanto que para a análise da convecção natural a correlação proposta por Churchill, Chu [11] para geometrias cilíndricas isotérmicas e estacionárias foi empregada.

Já para a região 2 descrita anteriormente como uma região de dissipação térmica na elaboração do modelo integral admitiu-se nessa região um coeficiente de dissipação de calor resultante $\left(h_{\text {Resultante }}\right.$ ) entre o coeficiente convectivo $(\bar{h})$ proveniente da convecção combinada ou simplesmente forçada, ou seja, $\left(\bar{h}_{\text {Total }}\right)$ ou $\left(\bar{h}_{\text {Forçado }}\right)$ dependendo da relação existente entre o número de Grashof (Ga) e número de Reynolds $(\mathrm{Re})$, mais o proveniente da radiação entre o rolo e ambiente ( $\left.h_{\text {Radiação Térmica }}\right)$. A (Equação 7$)$ descreve tal fato.

$$
h_{\text {Resultante }}=\bar{h}+h_{\text {Radiação Térmica }}
$$

$\mathrm{Na}$ qual o coeficiente convectivo $(\bar{h})$ foi determinado pela mesma metodologia e considerações descritas no equacionamento desse parâmetro para a região 1. Já o coeficiente proveniente da radiação térmica ( $h_{\text {Radiação Térmica }}$ ) baseou-se na (Equação 8).

$$
h_{\text {Radiação Térmica }}=\frac{\sigma \varepsilon_{\text {Rolo }}\left(T_{\text {sup-rolo }}{ }^{4}-T_{\infty}{ }^{4}\right)}{T_{\text {sup-rolo }}-T_{\infty}}
$$

* Contribuição técnica ao $35^{\circ}$ Seminário de Balanços Energéticos Globais e Utilidades e $29^{\circ}$ Encontro de Produtores e Consumidores de Gases Industriais, 13 a 15 de agosto de 2014, São Paulo, SP, Brasil. 


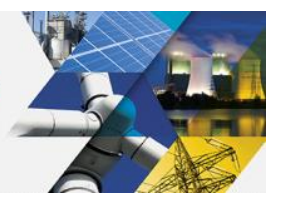

Equação na qual as temperaturas referentes à superfície do rolo $\left(T_{\text {sup-rolo }}\right)$ e da corrente livre $\left(T_{\infty}\right)$ foram definidas de modo análogo ao descrito quando do emprego dessas grandezas na região 1. Assim com base no equacionamento proposto para essa região, definiu-se então como condição de contorno para a mesma um coeficiente de transferência de calor resultante $\left(h_{\text {Resultante }}\right)$ entre o coeficiente convectivo $(\bar{h})$ e o coeficiente de transferência de calor por radiação

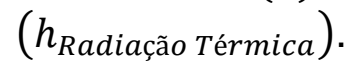

No que tange as regiões 3 e 4 descritas anteriormente como lado do mancal refrigerado e lado do mancal não refrigerado respectivamente, adotadas como condições de contorno para implementação computacional durante a etapa de criação do modelo integral, as mesmas foram admitidas como regiões adiabáticas. Hipótese essa baseada na existência de geração de calor local nos mancais proveniente da conversão de energia mecânica relativa ao movimento dos rolamentos em energia térmica (dissipação viscosa).

Por fim na configuração adotada para a região 5 , região na qual existe a refrigeração do sistema por meio do escoamento de água no interior de uma cavidade, a determinação do coeficiente convectivo médio $\left(\bar{h}_{\text {Refrigeração }}\right)$ existente foi empregada como a última condição de contorno a ser equacionada para a posterior inserção dessa variável na implementação computacional. Para isso o emprego da correlação proposta por Incropera et al. [6] para o cálculo do número de Nusselt e consequente determinação do $\left(\bar{h}_{\text {Refrigeração }}\right)$ para escoamento interno em geometria circular foi admitido.

\subsection{Aquisição de Dados}

A etapa de aquisição de dados empregada para determinação das condições de contorno propostas no modelo integral simplificado dos meios de transmissão de calor consistiu na determinação dos seguintes parâmetros: Temperatura da superfície do rolo, temperatura da superfície do tubo, vazão de trabalho do sistema de refrigeração, temperatura de entrada da água no sistema de refrigeração e temperatura de saída da água do sistema de refrigeração. A determinação da temperatura da superfície do rolo foi executada por meio de um mapeamento termográfico. $\mathrm{O}$ equipamento empregado para aquisição dos dados foi uma câmera infravermelha, fabricante: FLIR, modelo: E300, faixa de medição: $0^{\circ} \mathrm{C}$ a $500^{\circ} \mathrm{C}$, resolução $0,1^{\circ} \mathrm{C}$ representada pela (Figura 2).

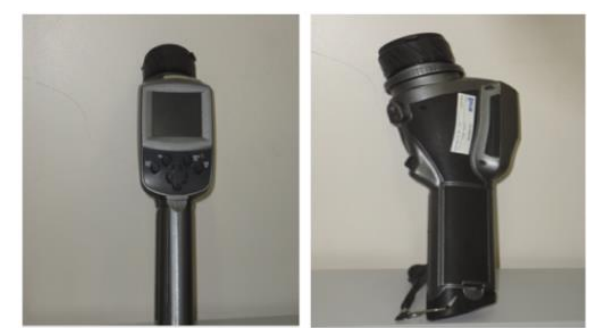

Figura 2: Câmera termográfica

Já para avaliação da temperatura da superfície do tubo, o procedimento adotado consistiu na realização de três ciclos de medições em que cada ciclo foi representado por 10 amostras. A temperatura final do tubo foi definida como a média aritmética das medições realizadas. O equipamento utilizado foi um pirômetro,

* Contribuição técnica ao $35^{\circ}$ Seminário de Balanços Energéticos Globais e Utilidades e $29^{\circ}$ Encontro de Produtores e Consumidores de Gases Industriais, 13 a 15 de agosto de 2014, São Paulo, SP, Brasil. 


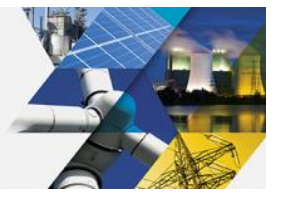

fabricante: Raytec, modelo: 2MSC, faixa: 200 a $1800{ }^{\circ} \mathrm{C}$, resolução: $1^{\circ} \mathrm{C}$, representado pela (Figura 3).

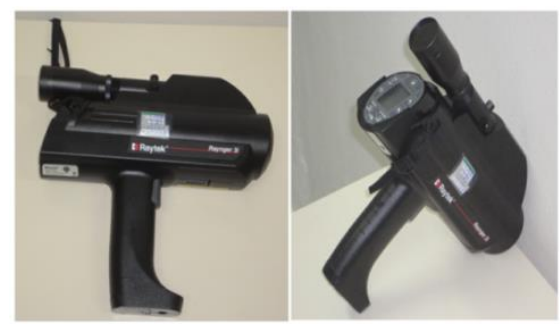

Figura 3: Pirômetro

A aquisição dos dados referentes a vazão volumétrica foi executada por meio de 3 ciclos de medições distintas cada um composto por 10 amostras. A vazão volumétrica final do sistema de refrigeração foi definida como a média aritmética das medições realizadas. O equipamento adotado para avaliação dessa grandeza foi um medidor de vazão, fabricante: Siemens, modelo: FUP 1010, resolução: 0,01 m³/h, conforme representa a (Figura 4).

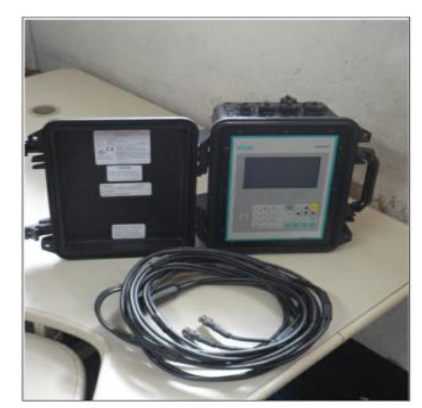

Figura 4: Medidor de Vazão

A respeito da temperatura de entrada da água do sistema de refrigeração para avaliação dessa grandeza foi utilizado um termômetro do fabricante: Wika, modelo: EN 13190, com resolução de $1^{\circ} \mathrm{C}$, apresentado pela (Figura 5), na qual foram realizados 3 ciclos de medições distintas cada um composto por 10 amostras. A temperatura final da entrada da água do sistema de refrigeração foi definida como a média aritmética das medições realizadas.

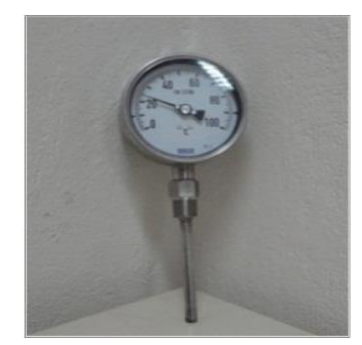

Figura 5: Termômetro

Por fim no que se refere à aquisição da temperatura de saída da água do sistema de refrigeração a avalição desse parâmetro foi executada por meio de um medidor de temperatura do tipo PT 100, representado pele (Figura 6), fabricante: ECIL, modelo: 1015, faixa: $0^{\circ} \mathrm{C}$ a $400^{\circ} \mathrm{C}$ em associação com um sistema supervisório de

* Contribuição técnica ao $35^{\circ}$ Seminário de Balanços Energéticos Globais e Utilidades e $29^{\circ}$ Encontro de Produtores e Consumidores de Gases Industriais, 13 a 15 de agosto de 2014, São Paulo, SP, Brasil. 


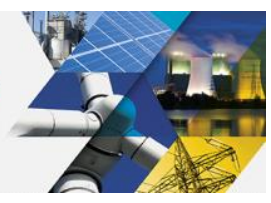

monitoramento contínuo. A avaliação dessa grandeza foi executada por meio de uma média aritmética dos dados coletados no supervisório.

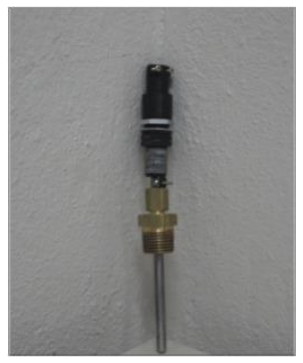

Figura 6: PT 100

\subsection{Implementação Computacional}

Para visualização dos perfis de temperatura no conjunto mecânico foi utilizada a dinâmica dos fluidos computacionais (CFD), no qual o problema foi tratado a partir de uma análise em regime estacionário, cujo domínio empregado durante a simulação foi um domínio sólido. O motivo para o emprego de tal domínio foi baseado no tratamento do problema a partir do ponto de vista de um problema de difusão térmica, de modo que para isso todas as condições de contorno adotadas foram descritas como condições do tipo WALL.

\section{RESULTADOS E DISCUSSÃO}

Com base no modelo integral proposto as seguintes condições de contorno apresentadas pela (Tabela 1) foram implementadas na análise computacional do problema.

Tabela 1: Condições de contorno empregadas na simulação computacional via CFD

\begin{tabular}{lll}
\hline Região térmica & Condição de contorno & Valores \\
\hline Fluxo de calor & $\dot{E}_{\text {entrada }}\left(\mathrm{W} / \mathrm{m}^{2}\right)$ & $73.284,79 \mathrm{~W} / \mathrm{m}^{2}$ \\
Dissipação térmica & $\bar{h}\left(\mathrm{~W} / \mathrm{m}^{2} \mathrm{~K}\right)$ e $T_{\infty}(\mathrm{K})$ & $28,10 \mathrm{~W} / \mathrm{m}^{2} \mathrm{~K}$ e $308 \mathrm{~K}$ \\
Mancal lado refrigerado & Superfície adiabática & - \\
Mancal lado não refrigerado & Superfície adiabática & - \\
Cavidade refrigerada & $\bar{h}_{\text {Refrigeração }}\left(\mathrm{W} / \mathrm{m}^{2} \mathrm{~K}\right)$ e $\overline{T_{m}}(\mathrm{~K})$ & $917,56 \mathrm{~W} / \mathrm{m}^{2} \mathrm{~K}$ e $310 \mathrm{~K}$
\end{tabular}

Condições de contorno essas, as quais inseridas na análise computacional via CFD resultaram no perfil de temperatura representado pela (Figura 7), vista superior e inferior do conjunto mecânico. Resultados esses no quais comparados ao resultado da análise termográfica apresentado na (Figura 8) demostra que a temperatura máxima, assim como a região de ocorrência da mesma e a temperatura média do conjunto são muito próximas. Fato este apresentado na (Tabela 2).

* Contribuição técnica ao $35^{\circ}$ Seminário de Balanços Energéticos Globais e Utilidades e $29^{\circ}$ Encontro de Produtores e Consumidores de Gases Industriais, 13 a 15 de agosto de 2014, São Paulo, SP, Brasil. 

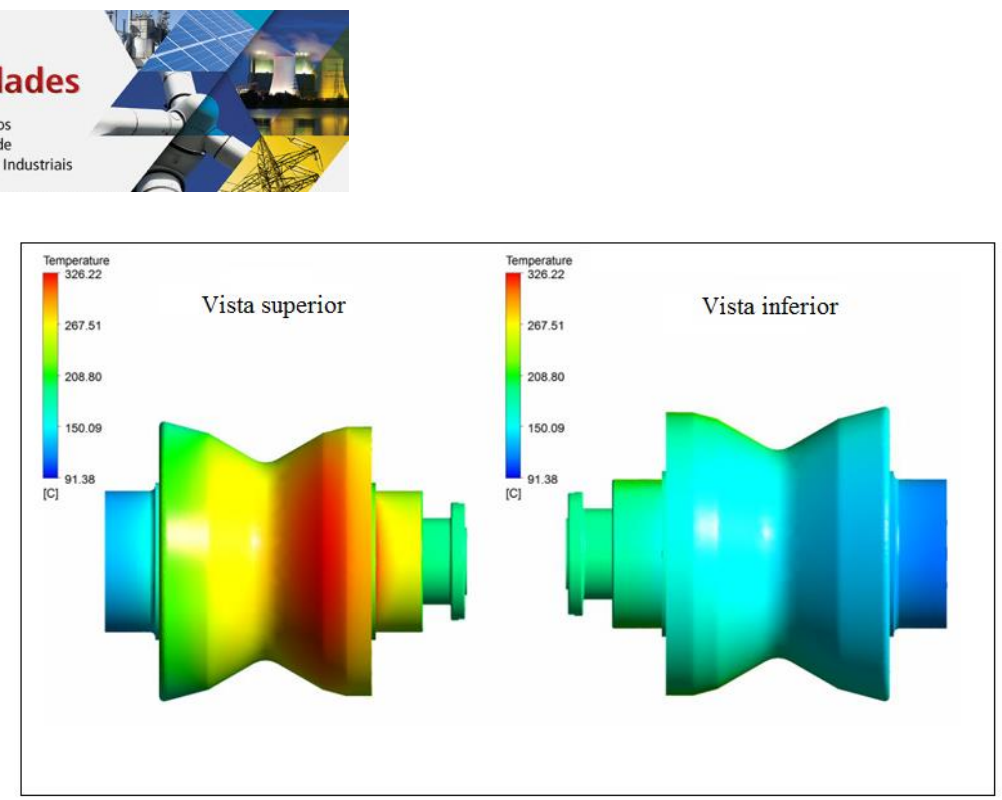

Figura 7: Vista superior e inferior do conjunto mecânico
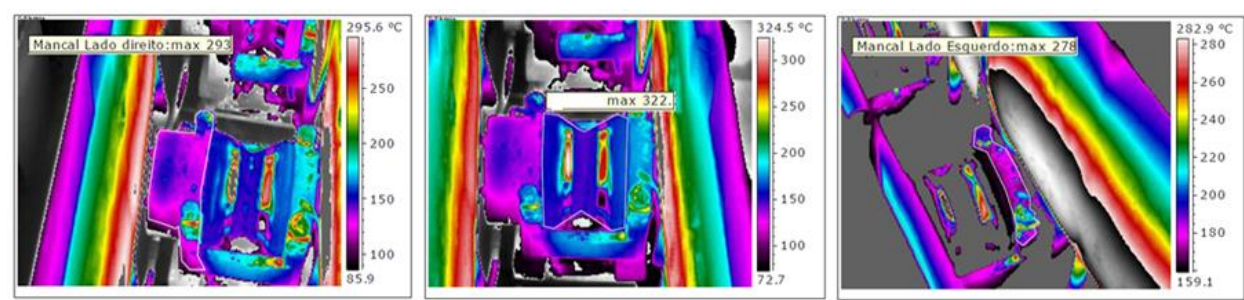

Figura 8: Imagens térmicas do conjunto mecânico (Câmera termográfica)

Tabela 2: Comparação termografia x simulação computacional

Análise Temperatura máxima $\left({ }^{\circ} \mathrm{C}\right)$

\section{Temperatura média $\left({ }^{\circ} \mathrm{C}\right)$}

\begin{tabular}{ccc}
\hline Termografia & 322.00 & 172.00 \\
Simulação computacional & 326.22 & 184.00 \\
\hline
\end{tabular}

Desse modo acredita-se que os resultados obtidos por meio da simulação computacional para a distribuição de temperatura do sistema podem de certo modo estar refletindo o verdadeiro perfil térmico desse conjunto na prática. Assim infere-se que os resultados obtidos na análise pertinente a distribuição de temperatura na pista interna do rolamento para ambos os lados dos mancais tanto o refrigerado como o não refrigerado, representados pelas (Figura 9) e (Figura 10), descrevam a temperatura real de contato da pista do rolamento com o fluido lubrificante (graxa).

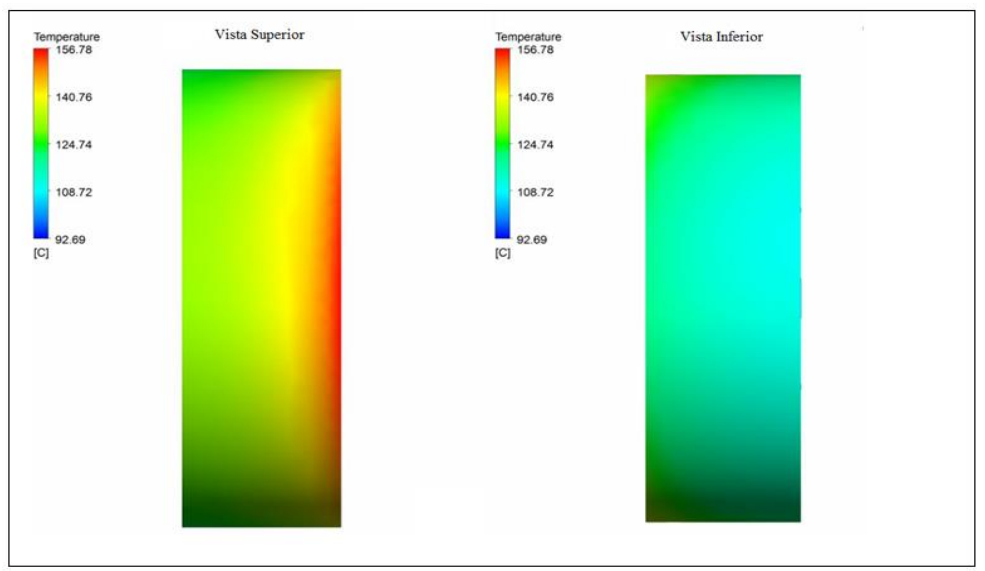

Figura 9: Pista interna do rolamento (Mancal lado refrigerado)

* Contribuição técnica ao $35^{\circ}$ Seminário de Balanços Energéticos Globais e Utilidades e $29^{\circ}$ Encontro de Produtores e Consumidores de Gases Industriais, 13 a 15 de agosto de 2014, São Paulo, SP, Brasil. 

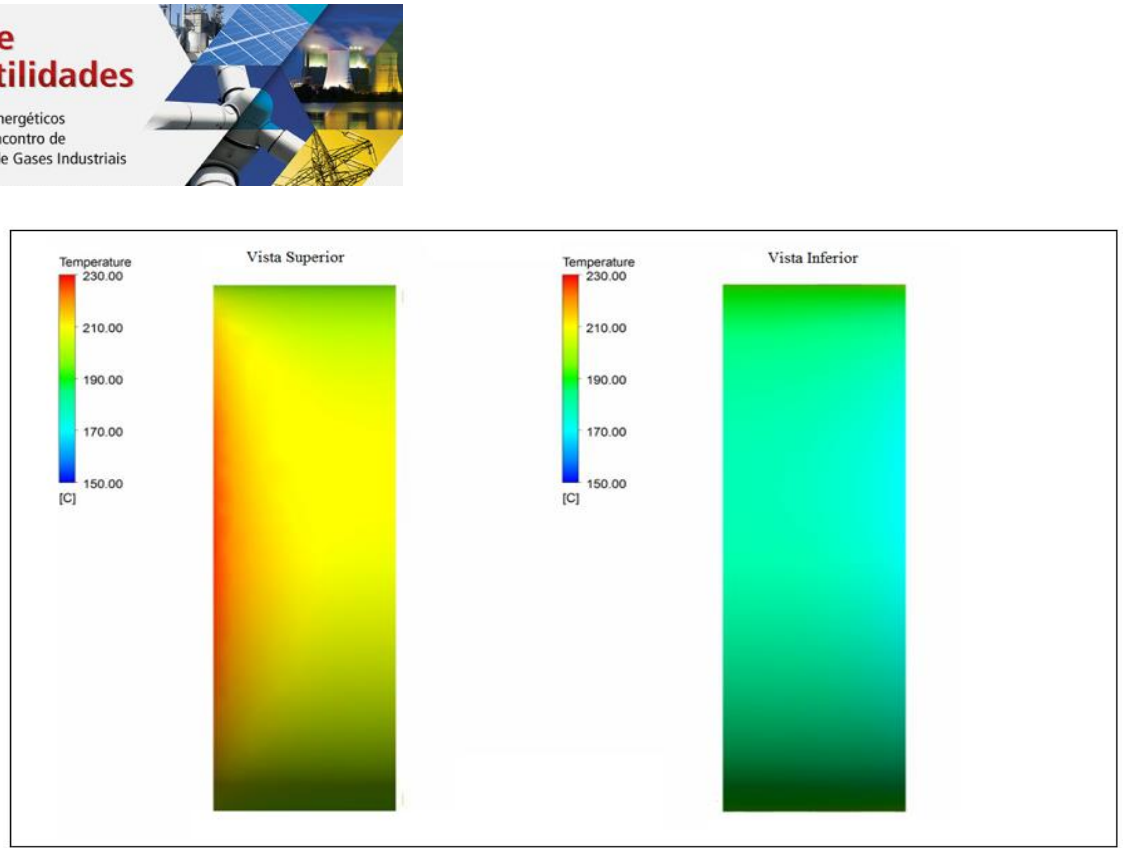

Figura 10: Pista interna do rolamento (Mancal lado não refrigerado)

A respeito das temperaturas médias obtida na pista interna do rolamento para os lados dos mancais $118,51^{\circ} \mathrm{C}$ (lado refrigerado) e $194,21^{\circ} \mathrm{C}$ (lado não refrigerado) observa-se, que na prática ambos os mancais possuem potencial para inicializar o fenômeno de ponto de gota. Potencial este referenciado pela presença de temperaturas estabelecida entre a faixa de temperatura para inicialização do fe nômeno para diversos tipos de graxas industriais como descrevem a (Tabela 3) e a (Tabela 4) respectivamente.

Tabela 3: Temperatura de ponto de gota graxas de Cálcio, Sódio, Complexas e Bário

\begin{tabular}{cc}
\hline Análise & Temperatura $\left({ }^{\circ} \mathbf{C}\right)$ \\
\hline Graxas de Cálcio & 70 a 120 \\
Graxas de Sódio & 120 a 200 \\
Graxas Complexas & $>260$ \\
Graxas de Bário & 180 a 260
\end{tabular}

Fonte: Lubrificantes e Lubrificação Industrial, 2006, p. 101.

Tabela 4: Temperatura de ponto de gota graxa de Lítio, Alumínio e Poliuréia

\begin{tabular}{cc}
\hline Análise & Temperatura $\left({ }^{\circ} \mathbf{C}\right)$ \\
\hline Graxas de Lítio & 180 a 250 \\
Graxas de Alumínio & 70 a 110 \\
Graxas de Poliuréia & 220 a 260
\end{tabular}

Fonte: Lubrificantes e Lubrificação Industrial, 2006, p. 101.

Em consideração ainda a distribuição de temperatura obtida na pista interna do rolamento observa-se que a temperatura máxima obtida no lado do mancal não refrigerado foi de $230^{\circ} \mathrm{C}$, enquanto que do lado refrigerado o resultado apresentado foi de $156,76^{\circ} \mathrm{C}$. O que assim descreve um aumento de temperatura de $46,72 \%$ quando da comparação entre o sistema não refrigerado para o refrigerado. Com base nos resultados do perfil térmico descrito para o conjunto mecânico em questão

* Contribuição técnica ao $35^{\circ}$ Seminário de Balanços Energéticos Globais e Utilidades e $29^{\circ}$ Encontro de Produtores e Consumidores de Gases Industriais, 13 a 15 de agosto de 2014, São Paulo, SP, Brasil. 
referente ao emprego de graxas complexas para a lubrificação do sistema, haja vista que a temperatura de inicialização do ponto de gota é superior a $260^{\circ} \mathrm{C}$. Já a segunda consiste na extensão do sistema de refrigeração para pontos mais próximos do lado do mancal não refrigerado.

\section{Agradecimento}

Aos professores Dr. Márcio Fonte Boa Cortez e Dr. Rudolf Huebner da Universidade Federal de Minas Gerais (UFMG) pelo apoio e ajuda na elaboração do estudo desenvolvido.

\section{REFERÊNCIAS}

$1 \quad$ NSK. Catálogo NSK rolamentos. [S.I:s.n].[entre 2005 e 2010].183 p.

2 Stouterau, Rodrigo Lima. Tribologia. [S.I.: s.n.], 2004. 179 p.

3 Carreteiro, Ronald P.; Belmiro, Pedro Nelson A. Lubrificantes e lubrificação industrial. Rio de Janeiro: Interciência, 2006.

4 Burmeister, Louis C. Convective heat transfer. 2. ed. New York: Johh Wiley e Sons, 1993.

5 Chevrom Brasil. Fundamentos de lubrificação. Rio de Janeiro:[s.n], 2005.

6 Incropera, Frank P. et al. Fundamentos de transferência de calor e massa. Rio de Janeiro: LTC, 2008.

7 Kreith, Frank; Bohn, Mark S. Princípios da transferência de calor.São Paulo: Thomson Learning, 2003.

8 Siegel, Robert; Howell John R. Thermal radiation heat transfer. [S.I]: McGraw-Hill, 1972.

9 Feingold, A; Gupta, K.G; New analytical approach to the evaluation of configuration factors in radiation from spheres and infinitely long cylinders. Journal heat transfer. 1970;92(1):69-76.

10 Churchill, S. W.; Bernstein, M. Journal heat transfer, 99, 300, 1977 apud Incropera, Frank P. et al. Fundamentos de transferência de calor e massa. Rio de Janeiro: LTC, 2008.

11 Churchill, S.W., and H.H.S. Chu, Int. J. Heat Mass Transfer, 18, 1049, 1975 apud INCROPERA, Frank P. et al. Fundamentos de transferência de calor e massa. Rio de Janeiro: LTC, 2008. 639 p.

* Contribuição técnica ao $35^{\circ}$ Seminário de Balanços Energéticos Globais e Utilidades e $29^{\circ}$ Encontro de Produtores e Consumidores de Gases Industriais, 13 a 15 de agosto de 2014, São Paulo, SP, Brasil. 